\title{
Low-coordination Topologies For Redundancy In Sensor Networks
}

\author{
Rajagopal lyengar \\ ECSE Department \\ Rensselaer Polytechnic \\ Institute \\ Troy, NY 12180 \\ iyengr@rpi.edu
}

\author{
Koushik Kar \\ ECSE Department \\ Rensselaer Polytechnic \\ Institute \\ Troy, NY 12180 \\ kark@rpi.edu
}

\author{
Suman Banerjee \\ CS Department \\ University of Wisconsin \\ Madison, WI 53706 \\ suman@cs.wisc.edu
}

\begin{abstract}
Tiny, low-cost sensor devices are expected to be failure-prone and hence in many realistic deployment scenarios for sensor networks these nodes are deployed in higher than necessary densities to meet operational goals. In this paper we address the question of how nodes should be managed in such dense sensor deployments so that the network topology formed by the active sensors is able to provide connected-coverage to the entire area of interest and at the same time increase the lifetime of the network. In particular, we propose and study distributed, low-coordination node wakeup schemes to efficiently construct multiple independent (node-disjoint) sensor network topologies to achieve good fault tolerance. We propose and evaluate different distributed, random and pattern-based wakeup policies for sensor nodes to construct connected-covered topologies. Through analysis and simulations we demonstrate that in dense sensor deployment scenarios, these policies can construct near-optimal topologies (within $2.7 \%$ of the optimal) with zero coordination between nodes, as long as location information is available at the individual sensor nodes. Based on these observations, we develop and evaluate a few simple distributed, wakeup based topology construction algorithms that can realize similar performance bounds in realistic sensor deployments, with varying node densities. These algorithms differ in terms of the required level of coordination and the use of sensor location information, and generate connected-covered topologies efficiently, with very low message-exchange overhead.
\end{abstract}

\section{Categories and Subject Descriptors}

C.2.1 [Computer-Communication Networks]: Network Architecture and Design

\section{General Terms}

Algorithms, Theory, Performance.

Permission to make digital or hard copies of all or part of this work for personal or classroom use is granted without fee provided that copies are not made or distributed for profit or commercial advantage and that copies bear this notice and the full citation on the first page. To copy otherwise, to republish, to post on servers or to redistribute to lists, requires prior specific permission and/or a fee.

MobiHoc'05, May 25-27, 2005, Urbana-Champaign, Illinois, USA.

Copyright 2005 ACM 1-59593-004-3/05/0005 ...\$5.00.

\section{Keywords}

Sensor Networks, Connectivity, Coverage, Reliability.

\section{INTRODUCTION}

Due to major technological innovations in recent years, development of tiny, low-cost sensor devices has become possible. Using a large number of such devices, it is today possible to construct a rich networked environment that can significantly enhance our interaction with the physical world. Networks of such sensor devices can be deployed in different environments for various data gathering purposes, e.g., environmental and weather monitoring, area survey before military deployment, monitoring of hazardous leaks in reactors, information gathering to plan relief operations at disaster sites, etc. In many of these sensor network applications, wireless transmission may be the only possible mode of communication. These networks may need to be deployed at short notice, or in hostile or inaccessible environments, and setting up a wired network in such situations is not feasible, or cost-effective. Therefore, in most sensor network applications, a sensor node would act both as a sensor and as a wireless transmitter (the sensor device may have separate sensing and transmitting units within itself).

In order for sensor networks to be cost efficient, it is expected that individual sensor nodes are relatively cheap, and hence prone to failures. Therefore, such sensors will need to be deployed conservatively in high densities to achieve a reasonable level of faulttolerance. In such scenarios, the number of operational sensor nodes in a region can be much higher than the minimum needed to perform the necessary functions. Moreover, limitations on the sizes of sensors puts constraints in terms of the battery energy, and the maximum transmission power (i.e., maximum transmission range). In order to extract a high operational lifetime of the network it is expected that only a subset of these nodes are activated at any given instant, while the rest are put to "sleep." By timesharing between different sets of such sensor nodes it is possible achieve a longer lifetime for the network. A number of recent proposals have used such an approach for increasing the lifetime of sensor networks, e.g. GAF [12], SPAN [10], STEM [14], AFECA [13], LEACH [11]. However the goal of these recent research activities has been to construct connected topologies only. In contrast, the work described in this paper addresses the problem of connectedcoverage, i.e., not only does the set of active sensors form a connected subset, the combined sensing range covers the entire (or a large portion of the) region of interest. Additionally our proposed schemes requires minimal coordination between the sensor nodes themselves while achieving these goals.

A related work by Gupta et. al. [9], with objective similar to 
ours, demonstrated how to construct a single connected-covered set of sensor nodes. They propose a centralized algorithm that finds a connected-covered set within a $O(\log n)$ approximation factor of the optimal connected-covered set. Subsequently they propose a distributed variant of this algorithm that requires a moderate amount of coordination between the nodes. Our work differs from their approach in the following ways: (1) Our objective is to find not one, but multiple connected-covered sets in a given network deployment. In particular our goal is to maximize the number of such independent (node-disjoint) connected-covered sets which can be used to provide greater redundancy and fault-tolerance. Note here that two independent (or node-disjoint) topologies do not have any sensor node in common. (2) We assume that the deployment of sensor networks is dense which is practical for many deployment scenarios. Under this assumption we present distributed algorithms that finds each connected-covered set whose size is within a small constant factor of the optimal connected-covered set (i.e., about $2.7 \%$ ) in comparison to the $O(\log n)$ bound in [9]. (We should, however, add that authors in [9] make no density assumptions and hence their proposed algorithm provides the best known theoretical bounds for sparse deployment scenarios.) (3) Our approaches requires minimal or no coordination between the sensor nodes.

\subsection{Wakeup based Topologies: Goals and Con- straints}

In order to achieve improved network lifetime, we define policies that dictates which sensor nodes should be powered on (or "woken up" or "activated") at a given instant. More specifically, a sensor node should be powered on only when activating the sensor node enhances sensor network functionality, avoiding redundant activation wherever possible. However, the wakeup policy should ensure that at any instant of time, the network topology formed by the sensors that are active at that time satisfy certain performance criteria. In this paper, we address the problem of constructing topologies that satisfy such criteria while minimizing the number of active sensors. Since this topology construction is effected through an appropriate choice of wakeup (or activation) strategy, we will refer to the proposed approaches both as topology construction strategies as well as node wakeup (or node activation) strategies.

\subsubsection{Coverage and Connectivity}

In developing effective wakeup based topologies, two metrics are of prime importance:

Coverage: Each sensor device typically has a physical sensing range within which it is able to perform its operation. An effective node activation scheme should ensure that the entire physical space of interest (or a large fraction of it) is within the sensing range of at least one of the active sensors.

Connectivity: Each sensor has a maximum physical range for direct communication, which determines its connectivity. It is typically more energy efficient to aggregate all the sensor data at a few specific wireless nodes (gateways) from where the data can be uploaded to the remotely located monitoring station. Therefore, another deployment objective is to ensure that all the sensor nodes (or a large fraction of them) are connected to some gateway, possibly through some multi-hop path.

Sensor network topologies need to meet both these requirements of coverage and connectivity. These two important metrics - coverage and connectivity - can be nicely combined in the notion of connected-coverage, as defined below.

Connected-coverage: Any point in the region of interest is provided connected coverage if it is covered by (i.e., within the sensing range of) an active sensor node that is connected to (i.e., able to communi- cate to, possibly using a multi-hop path comprising of other active sensors) to a gateway node (Figure 1). Thus the gateway nodes can monitor (i.e., collect data from) only the region that is provided connected-coverage. In other words, the area covered by a sensor that is not connected to any gateway cannot be monitored, since the data from that area cannot reach the gateway. Therefore, the topology formed by the active sensors should be done in such a way that the entire region of interest, or a large fraction of it, is provided connected-coverage.

\subsubsection{Topology Construction Objectives}

Using the notion of connected-coverage as described above, our node wakeup based topology construction objective can be cast as an optimization problem, defined as follows. At any instant of time, we want to power on the sensor nodes in such a way such that the resulting sensor network formed by the active sensors provides connected-coverage to the entire region of interest by using a minimum number of active sensors. Henceforth, we refer to topologies that provide connected-coverage to the entire region of interest as connected-covered topologies (or cc-topologies, in short). Thus the goal of node wakeup policies is to construct cc-topologies by using the minimum number of sensors. Also, the efficiency of any wakeup based cc-topology construction algorithm is thus measured according to the number of sensor nodes (per unit area) required by the algorithm to achieve connected-coverage.

Note that our objective of minimizing the number of active sensors (subject to the constraints on connected-coverage) at any time is directly correlated with that of maximizing the lifetime of the overall sensor network. A sensor network with a high degree of redundancy can typically be decomposed into multiple independent (node-disjoint) network topologies providing connected-coverage to the entire region of interest. The lifetime of the overall sensor network can then be enhanced by time-sharing between these topologies, powering on only one of these topologies at any given time. Clearly, a larger number of such topologies would typically imply a longer network lifetime. Minimizing the number of sensors in these topologies will typically result in a larger number of such topologies to be formed from a same set of nodes, thereby increasing the lifetime of the network.

\subsubsection{Solution Requirements}

The size of the sensor network can be very large both in terms of the vastness of the deployment region and the number of deployed sensors. Therefore, for practical viability, a wakeup based topology construction algorithm must be distributed in nature. Moreover, it is also desirable that the algorithm be such that an individual sensor node is able to decide whether to remain awake or not based on local information exchange. Sensor nodes are typically severely resource-constrained, and this implies that the complexity of computation and information exchange in the topology construction algorithm should be very low. The solution approaches that we propose and study satisfy all these requirements.

Note that the connectivity and coverage radii ( $r$ and $\rho$, respectively) of a sensor node can be different, and the exact description of the solutions proposed in this paper and their analysis depend on the $\frac{r}{\rho}$ ratio. However, for the sake of definiteness and the simplicity of exposition, most of our discussion in this paper focuses on the case when $r=\rho$, although we occasionally stray for a brief discussion on the case when $r \neq \rho$. However, it is worth noting here that our solution approaches, as well as our analysis, can be adapted to scenarios where $r$ and $\rho$ are unequal. 


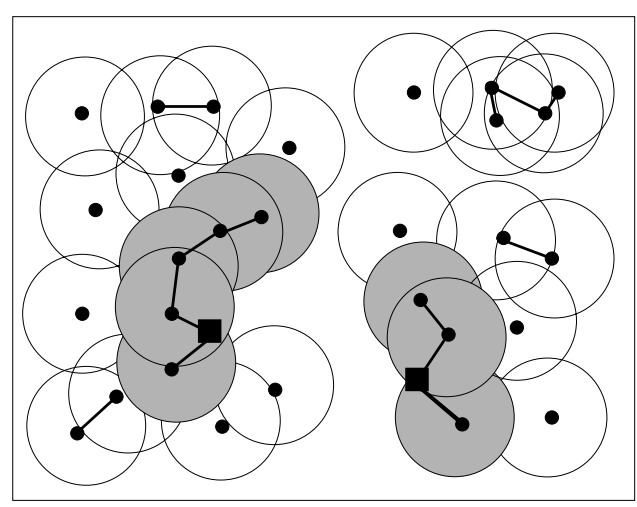

(a) $\rho=r=1$ unit

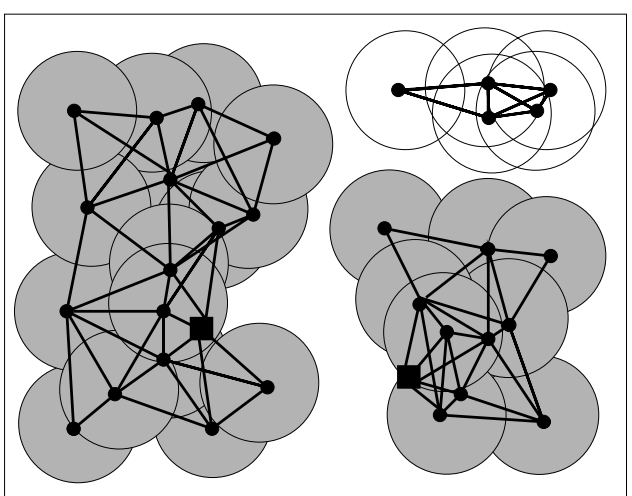

(b) $\rho=\frac{r}{2}=1$ unit

Figure 1: Connected-coverage. (The shaded region represents the connected-covered area. Each sensor is assumed to have circular coverage and connectivity patterns. Each sensor is modelled as a disk of coverage-radius $\rho$, and the two gateways are represented by the squares. The node positions in both (a) and (b) are the same. The edges between the nodes define the connectivity graph. An edge exists between two nodes iff the distance between them is no greater than communication-radius $r$.

\subsection{Contributions of this Work}

The main contributions of this work can be summarized as follows. We propose and evaluate distributed, low-coordination node activation/wakeup methods for efficient cc-topology construction in dense sensor networks. For such dense sensor deployments these distributed algorithms provide the following guarantees:

- A purely random wakeup based algorithm (with zero coordination requirements between nodes) is able to construct a cc-topology whose size is within a constant factor (3-4 times) of the optimal cctopology.

- Our proposed patterned wakeup based algorithms (which require location information and zero coordination between nodes) is able to construct a cc-topology whose size is within a small constant factor $(2.7 \%)$ of the optimal cc-topology.

Based on the intuition of these above algorithms, we propose some simple but practical variants (requiring minimal node coordination and interaction) that provide comparable performance for practical network deployments ranging from low density to high density of nodes. Through detailed simulations we show that these practical distributed algorithms are efficient across a large range of node deployment densities. In particular we believe that our simple, constrained breadth-first-search based algorithm, which does not require nodes to have location information and provides nearoptimal performance even in a realistic network environment is most suited in wireless sensor networks. For example, the number of independent cc-topologies (that can be used for timesharing) found by this algorithm is also within a constant factor of a loose upper-bound of the maximum number of independent cctopologies present (within $70 \%$ of this loose upper bound). The development of this algorithm is motivated by the theoretical analysis developed in this paper.

\subsection{Roadmap}

In Section 2, we state and prove a lower bound on the optimal number of sensors (per unit area) required for connected-coverage; this lower bound will be used subsequently as a benchmark against which different solutions are compared. In Section 3, we study the efficiency of random-wakeup and patterned-wakeup based cctopologies for dense sensor networks. Finally, in Section 4, we describe a few wakeup based cc-topology construction strategies that can be implemented in a practical setting with moderately dense sensor deployment, and evaluate their performance through simulations. We outline the related literature in Section 5 and conclude in Section 6.

\section{A LOWER BOUND ON NODE DENSI- TIES FOR CONNECTED-COVERAGE}

In this section, we derive a lower bound on the efficiency of the optimal cc-topology. More specifically, we obtain a lower bound on the spatial density of nodes (spatial density is the number of nodes per unit area) required by any topology that provides connectedcoverage to an area of interest. In Section 3.2, we show that this bound is fairly tight. This bound will be used in subsequent sections to evaluate the performance of various topology construction strategies that we study. Intuitions obtained from the derivation of this bound will also be used in Section 4 in developing a practical node wakeup policy later in this paper.

For the ease of analysis, we assume that the region that is to be provided connected-coverage is the entire two-dimensional plane. Assume that there is a single gateway node placed anywhere in the plane. We assume a circular coverage pattern, with a coverage radius $\rho$. We also assume a circular connectivity pattern with a connectivity radius $r$ (i.e., a node is able to communicate with any other node if it is within a distance $r$ from itself, otherwise not). For simplicity of analysis, we assume $r=\rho$. In order to provide connected-coverage to the entire two-dimensional plane, we clearly need an infinite number of sensor nodes. Thus the appropriate optimization metric in this case is the spatial density, i.e., the number of nodes required per unit area, rather than the total number of nodes. In the following theorem, $O P T$ represents the optimal topology that provides connected-coverage to the entire 2-d plane with the minimum density of nodes.

Theorem 1. Let $d_{O P T}$ represent the density of nodes required by the optimal topology $(O P T)$ providing connected-coverage to the entire 2-d plane. Then

$$
d_{O P T} \geq \frac{0.522}{r^{2}} .
$$

Proof. Assume a square region of dimensions $D \times D$ in which is provided connected-coverage by a topology of $n$ sensor nodes. 


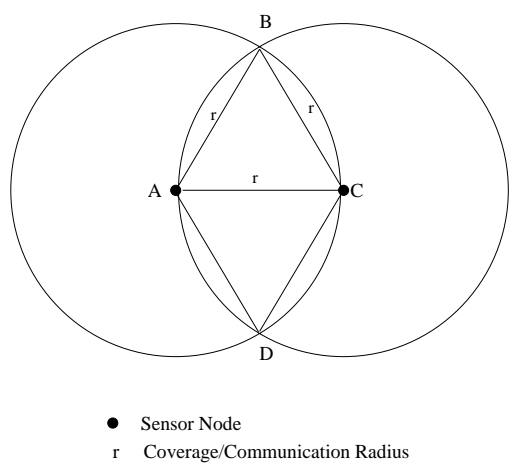

Figure 2: Overlap of area between two connected nodes.

By our definition of connected-coverage, these $n$ nodes must be connected. Let us traverse the connectivity graph of these $n$ nodes using a breadth-first or depth-first search technique. The first node traversed covers an area of $\pi r^{2}$. Each subsequent node effectively covers an area lesser than this since there must be some overlap in coverage areas with nodes already traversed. The minimum overlap scenario is represented in Figure 2. Here, the area in-between the curves BAD and BCD represents the minimum overlap. Simple geometric calculations show that the area of this overlap region is $\left(\frac{2 \pi}{3}-\frac{\sqrt{3}}{2}\right) r^{2}$. Therefore, the new area (area that is uncovered by previously traversed nodes) covered by the second, third, ... nodes is at most $\pi r^{2}-\left(\frac{2 \pi}{3}-\frac{\sqrt{3}}{2}\right) r^{2}=\left(\frac{\pi}{3}+\frac{\sqrt{3}}{2}\right) r^{2}$.

Hence,

$$
\begin{aligned}
D^{2} & \leq \pi r^{2}+(n-1)\left(\frac{\pi}{3}+\frac{\sqrt{3}}{2}\right) r^{2} \\
& \leq \pi r^{2}+1.914(n-1) r^{2} .
\end{aligned}
$$

We can rearrange the terms in the above equation to obtain

$$
\frac{n}{D^{2}} \geq \frac{1-\frac{(\pi-1.914) r^{2}}{D^{2}}}{1.914 r^{2}} .
$$

In the limit as $D$ goes to infinity, the above becomes

$$
\begin{aligned}
d_{O P T} & \geq \frac{1}{1.914 r^{2}} \\
& \geq \frac{0.522}{r^{2}} .
\end{aligned}
$$

The above theorem states that no topology can guarantee connectedcoverage to the entire $2-\mathrm{d}$ place with a node density lower than $\left(0.522 / r^{2}\right)$. For finite regions that are much larger in size compared to the sensor connectivity and coverage radii, this lower bound will hold to a fairly close degree.

\section{ALGORITHMS FOR CC-TOPOLOGIES IN DENSE SENSOR NETWORKS}

We now examine efficient algorithms for cc-topology construction in an idealized, dense sensor network. In this idealized scenario, we define a deployment to be dense if there is a sensor node close to any given location in the region of interest. While such a deployment may not always be practical, it allows us to provide some performance guarantees in presence of high densities as well as help us build intuition for more practical algorithms that apply to networks that do not require such density assumption (presented in Section 4). We evaluate the performance of different wakeup schemes experimentally using the following simulation setup. The region of interest, assumed to be a square, is approximated by a dense square grid of size $1000 \times 1000$. The sensor network is assumed to be very dense, and therefore, we assume that there is a sensor node at each grid point. There is also a single, randomlylocated gateway node.

Our algorithms in this section constructs a single cc-topology in such dense networks and analyzes the efficiency of such a cctopology with respect to the size of the optimal cc-topology. When we explore the practical variants of these algorithms (in Section 4) we will discuss how they can be recursively applied to the remaining set of sensor nodes to obtain our desired objective of creating multiple, independent cc-topologies.

\subsection{Random-Wakeup based CC-Topologies}

We first characterize the efficiency of cc-topologies where individual sensor nodes are woken up in a purely random manner. In the random-wakeup based topologies that we study in this section, once the number of nodes to be woken up is determined, the nodes are chosen from the area of interest uniformly at random. Such a strategy is closely approximated by a distributed algorithm where each individual sensor nodes wakes itself independently with a fixed probability (appropriately chosen). Such a distributed wakeup procedure requires no information (about location, local topology etc.) to be maintained at the sensor nodes, and can be implemented with zero coordination. For a uniformly deployed dense sensor network, such a distributed wakeup algorithm also yields performance nearly identical to that of the (more centralized) patterned strategies considered in this section.

We choose a number of sensors to be woken up $(N)$, and wake up $N$ sensors uniformly at random from the square grid considered. Figure 3 shows the evolution of the connected-covered area fraction as a function of the spatial density of active nodes, $\lambda\left(=N / D^{2}\right.$, where $D=1000$ ), for different values of coverage/connectivity radius $r$ (coverage and connectivity radii are assumed to be the same in this case). From the plot it is clear that a large fraction of the region of interest can be covered by sensor deployments in moderate densities. However to guarantee perfect coverage, the required density of nodes increases drastically. For example, with $r=10$, we can achieve $90 \%$ connected-coverage with a spatial density of 0.0095. However, the density required for complete connectedcoverage is about 0.014 . Therefore, for the sake of deployment efficiency in our practical algorithms in Section 4 we will limit our target connected-coverage to about $90 \%$ of the entire area.

Figure 4 plots similar results for the case when the connectivity radius $(r)$ and coverage radius $(\rho)$ are different.

From the plots, observe that the connected-covered area fraction shows a sharp transition, i.e., there is a certain threshold where the connected-covered area "shoots up" abruptly. For the case of $r=\rho$ (Figure 3), the value of this transition point is calculated empirically to be $\frac{1.45}{r^{2}}$, as shown in Figure 5. Interestingly, this threshold is very close to the empirically obtained critical density for continuum percolation of disk-graphs [8]. This abrupt transition in the connected-covered area is due to the fact that once the active node density reaches this threshold, connectivity of the network shows a "phase transition" and almost the entire network becomes connected.

From Figure 3, also note that the spatial density of active nodes required for providing connected-coverage to a significant fraction of the overall area is significantly larger than the lower bound in Theorem 1. For example, the transition point of $\frac{1.45}{r^{2}}$, is about 2.68 times the bound of $\frac{0.522}{r^{2}}$. It can also be observed that the 


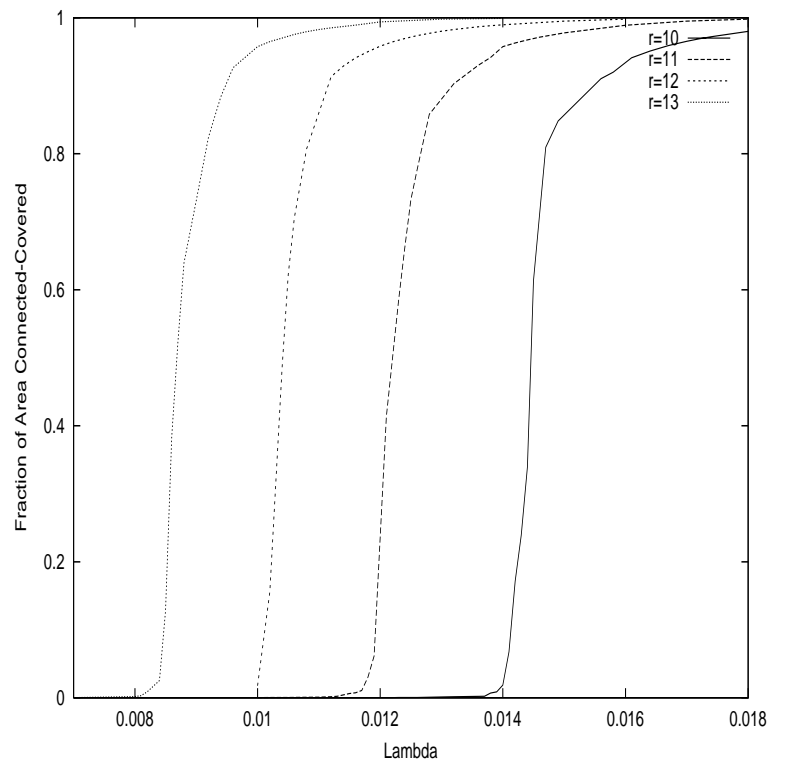

Figure 3: Connected-covered area fraction as a function of the active node density (as $r$ varies)

connected-covered area fraction at the bound value is nearly zero. Now consider the Figures 6, 7 and 8, which show the active node density required for attaining $80 \%, 90 \%$ and $99 \%$ connected-coverage, respectively, as a function of the different values of $r(=\rho)$. As the figures show, the plots are closely approximated by the curves $\frac{0.15}{r^{2}}$, $\frac{0.16}{r^{2}}$ and $\frac{0.19}{r^{2}}$, respectively. Comparing with the lower bound, we observe that the random wakeup scheme is able to provide connectedcoverage to almost the entire network with a density of about 3 - 4 times the lower bound on the optimum.

\subsection{Patterned-wakeup based CC-Topologies}

We now consider several wakeup policies that wakeup nodes to form a well-defined and regular pattern and use these nodes to construct cc-topologies in the area of interest. Our analysis reveals that there exists some patterns (e.g., STR as discussed later) that is able to achieve an efficiency within $2.7 \%$ of the optimal cc-topology construction. The formation of these pattern-based cc-topologies would require each sensor node to know its geographic location. If such information is available, these pattern-based cc-topologies can be realized in a fully distributed manner in dense networks, with no additional coordination.

By our density assumption, there is a sensor node located close to any chosen location. In other words, it is possible to activate a sensor at any specific location of our choice. Again, for the ease of analysis, we consider the case when the region of interest is the entire 2-d plane, although the results would hold very closely for large finite-size regions as well. We first consider the case where $r=\rho$; we comment on the case when $r$ and $\rho$ are different later in this section. We assume that there is single gateway node whose connectivity radius is $r$; in all of the topologies described below, the gateway can be placed anywhere in the region of interest.

\subsubsection{Square-grid based CC-Topology.}

Imagine laying out a square grid on the area of interest, where the spacing between adjacent grid lines is $r$. In the square-grid based cc-topology, the sensor nodes located at the grid-points of this square grid are woken up, as shown in Figure 9. Note that each node is in the transmission range of four other nodes, and all

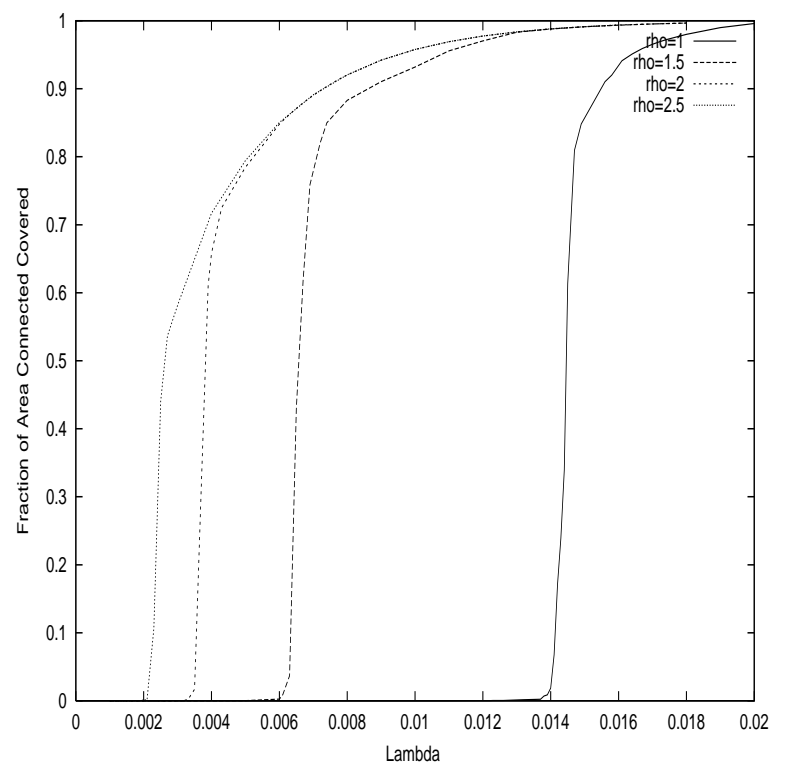

Figure 4: Connected-covered area fraction (as a function of the active node density) as $\frac{r}{\rho}$ varies ( $r$ is kept fixed at 1 , and $\rho$ varies)

nodes are connected to one another (possibly through multi-hop paths). Also note that the entire pattern can be viewed as a collection of squares, and each of these squares are covered (recall that $\rho=r)$. Therefore, this pattern of active sensor nodes guarantees connected-coverage to the entire 2-d plane.

Note that for a $D \times D$ square region $(D \gg r)$, there are roughly $(D / r)$ grid lines in both horizontal and vertical directions. Therefore, there are roughly $\left(D^{2} / r^{2}\right)$ nodes in this region, which leads to the following observation:

Observation 1. The spatial density of nodes in the square-grid based topology, $d_{S Q R}$, is given by:

$$
d_{S Q R}=\frac{1}{r^{2}}
$$

A comparison with Theorem 1 shows that the density of this cctopology is about 2 times the lower bound on the spatial node density required for connected-coverage.

\subsubsection{Hexagonal-grid based CC-Topology.}

The hexagonal-grid based cc-topology is constructed as follows. First, let us tile the entire 2-d plane with hexagons of side $r$ in the manner shown in Figure 10. Then, wake up nodes at the alternate vertices of the hexagons, and one at center of each hexagon. Note that each node is in the transmission range of three other nodes, and all nodes are connected to one another (possibly through multi-hop paths). Also note that each of the hexagons is completely covered. Therefore, this pattern guarantees connected-coverage to the entire 2-d plane. Note here that if we only placed the nodes at the centers of the hexagons (and not at the vertices), the entire plane would still have been covered. This is, in fact, the optimal topology for ensuring coverage [4], if we do not take connectivity into account. However, in such a topology, all the nodes are isolated, and therefore it does not guarantee connected-coverage. 


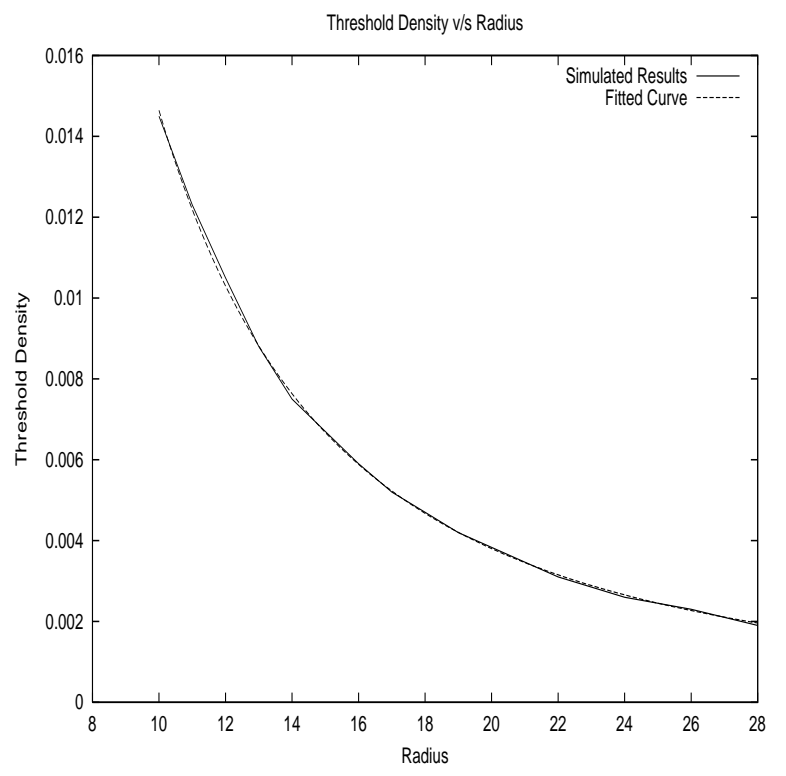

Figure 5: Threshold Active node density (as $r$ varies). The fitted curve is $\frac{1.45}{r^{2}}$.

Observation 2. The spatial density of nodes in the hexagonalgrid based topology, $d_{S Q R}$, is given by:

$$
d_{H E X}=\frac{0.769}{r^{2}} .
$$

The above result can be easily derived in the following way. Consider $D \times D$ square region $(D \gg r)$. Note that the cc-topology can be viewed as a collection of horizontal lines of active nodes, such that the spacing between adjacent horizontal lines varies alternately as $r$ and $(r / 2)$. Therefore, there are roughly $2 D /\left(r+\frac{r}{2}\right)=\frac{4 D}{3}$ horizontal lines in the $D \times D$ region. Also, since the spacing between adjacent active nodes in each horizontal line is $\sqrt{3} r$, there are roughly $\frac{D}{\sqrt{3} r}$ active nodes in each horizontal line. Therefore, there are approximately $\frac{4}{3 \sqrt{3}} \frac{D^{2}}{r^{2}} \approx 0.769 \frac{D^{2}}{r^{2}}$ active nodes in the region.

A comparison with Observation 1 and Theorem 1 shows that this topology guarantees connected-coverage with a lower density than the square-grid based topology, and the node density of this cctopology is roughly 1.5 times the lower bound.

Comparing the efficiencies of the two grid-based cc-topologies just considered with the randomly constructed topology discussed in Section 3.1, we observe that the grid-based topologies are considerably more efficient than the latter. This demonstrates that for dense networks, the efficiency of cc-topologies can be improved significantly if location information is available to the sensor nodes.

\subsubsection{Strip based CC-Topology.}

Next we describe a topology that guarantees connected-coverage with near-minimum spatial density of nodes. First, consider a string of active sensor nodes arranged in a line, such that the spacing between adjacent active nodes is $r$. Therefore, these nodes form a single connected component. Panel (a) in Figure 11, shows such a string of nodes, along with their connectivity/coverage radii; we call this string of active nodes a strip. Next imagine arranging these strips horizontally in the following manner. For every even integer $k$, place a strip oriented horizontally such that one of the nodes in the strip is positioned at $\left(0, k\left(\frac{\sqrt{3}}{2}+1\right) r\right)$. Also, for every odd inte-

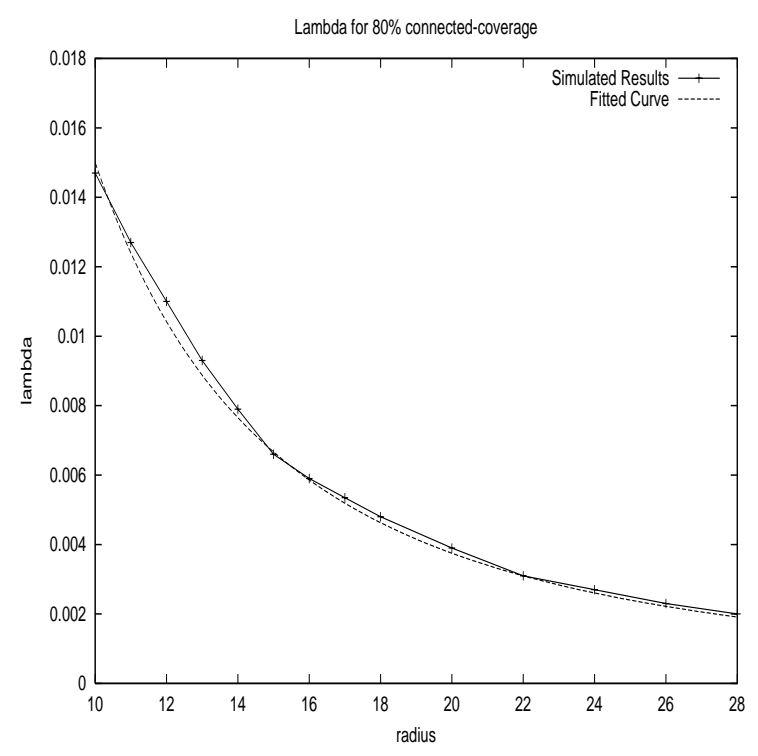

Figure 6: Active node density for $\mathbf{8 0 \%}$ connected-covered area fraction (as $r$ varies). The fitted curve is $\frac{1.5}{r^{2}}$.

ger $k$, place a strip horizontally such that the point $\left(\frac{r}{2}, k\left(\frac{\sqrt{3}}{2}+1\right) r\right)$ is a center of a node in the strip. This is shown in Panel (b), Figure 11. Next place some nodes vertically in the following way. For every odd integer $k$, place two nodes, at $\left(0, k\left(\frac{\sqrt{3}}{2}+1\right) r \pm\right.$ $\left.\frac{\sqrt{3}}{2} r\right)$. These nodes, along with their connectivity/covergae radii, are shown by the shaded disks in Figure 11. Let us denote the entire disk pattern of nodes (as shown in Figure 11) as $S T R$.

It can be verified that the collection of the horizontal strips cover all points in the plane. Note that in the absence of the vertical string of nodes, the nodes belonging to one strip is not connected to the nodes of another strip. The purpose of the vertical string of nodes is to connect the horizontal strips, and thus ensure connectivity between all nodes. Therefore the topology $S T R$ provides connected-coverage to the entire 2-d plane.

Observation 3. The density of nodes in the strip-based topology, $d_{S T R}$, is given by:

$$
d_{S T R}=\frac{0.536}{r^{2}}
$$

The above result can be derived in the following way. From the description above, it follows that the spacing between adjacent horizontal strips is $\left(\frac{\sqrt{3}}{2}+1\right) r$. Therefore, in a $D \times D$ square region $(D \gg r)$, there will be roughly $\frac{D}{\left(\frac{\sqrt{3}}{2}+1\right) r}$ strips. The spacing between adjacent nodes in each strip being $r$, there are about $(D / r)$ nodes in each strip. Therefore, the total number of active nodes due to the horizontal strips is $\frac{1}{\frac{\sqrt{3}}{2}+1} \frac{D^{2}}{r^{2}} \approx 0.536 \frac{D^{2}}{r^{2}}$. Note that the contribution due to the vertically string of active nodes will be proportional to $D$ (and not $D^{2}$ ), and is therefore negligible in the limit as $D \rightarrow \infty$.

Combining Theorem 1, the Observations 1, 2 and 3, and he fact that $d_{S T R} \geq d_{O P T}$, we obtain the following results: 


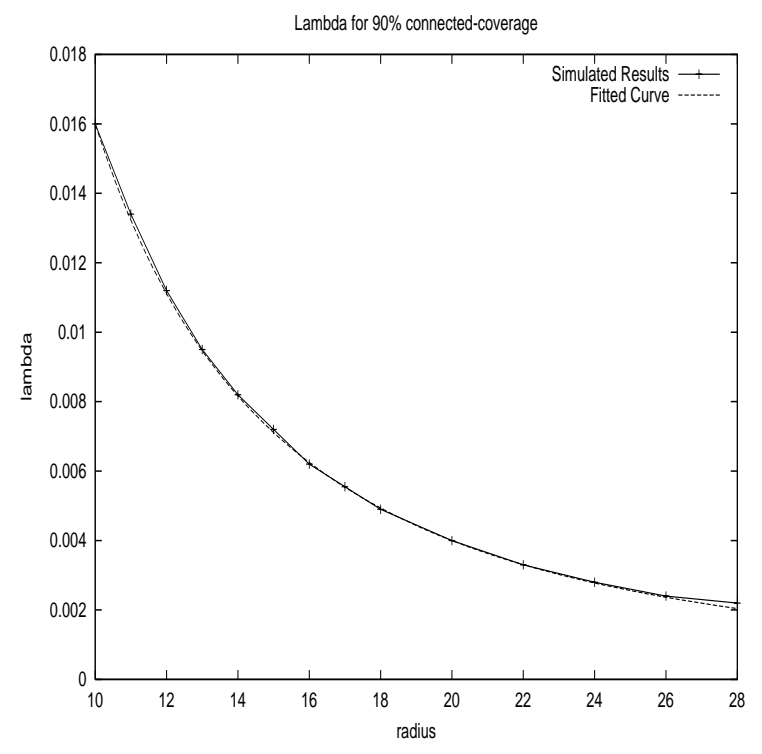

Figure 7: Active node density for $90 \%$ connected-covered area fraction (as $r$ varies). The fitted curve is $\frac{1.6}{r^{2}}$.

Corollary 1 . The following inequalities hold:
(i) $1 \leq d_{S T R} / d_{O P T} \leq 1.027$.
(ii) $1.434 \leq d_{H E X} / d_{O P T} \leq 1.473$.
(iii) $1.865 \leq d_{S Q R} / d_{O P T} \leq 1.916$.

The above result shows that the cc-topology $S T R$ is within $2.7 \%$ of the optimal solution, whereas the $H E X$ and $S Q R$ cc-topologies have at least $43.4 \%$ and $86.5 \%$ redundancy, respectively. Also note that since the solution $S T R$ is so close to the lower bound on the optimal, it shows that the bound stated in Theorem 1 is fairly tight.

\subsection{The case of unequal coverage and connec- tivity radii}

In general, the connectivity radius $(r)$ and the coverage radius $\rho$ can be different from one another. It is possible to extend the pattern based topologies discussed above so that they provide connectedcoverage when $r$ and $\rho$ are unequal. For the case $r \leq \rho$, a natural extension of STR yields performance bounds at least as good as that for the case of $r=\rho$. When $r$ is significantly larger than $\rho$, then a slight modification of the HEX topology works well. To visualize this modified HEX cc-topology, imagine tiling the entire 2-d plane with hexagons of side $\rho$, and then waking up a node at the center of each hexagon. In fact, this 'honeycomb' structure can be shown to be the optimal cc-topology if $r \geq \sqrt{3} \rho$.

\section{WAKEUP BASED INDEPENDENT CC-TOPOLOGY CONSTRUCTION}

In the random and patterned wakeup based topologies we have studied and analyzed so far, the sensor network was assumed to be so dense that there is at least one node close to any chosen location. This implies that we can wake up nodes at any location of our choice. However, this assumptions may not be true in practice. In this section, therefore, we define algorithms that can be used to construct efficient cc-topologies in more realistic settings, including moderately dense sensor networks. The results of the previous sections are however useful in this context; three of the algorithms

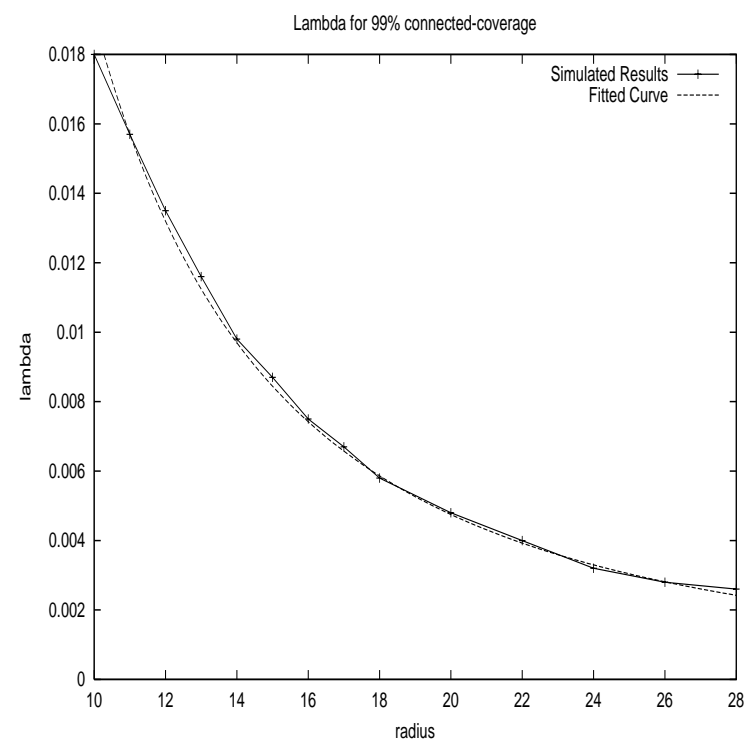

Figure 8: Active node density for $99 \%$ connected-covered area fraction (as $r$ varies). The fitted curve is $\frac{1.9}{r^{2}}$.

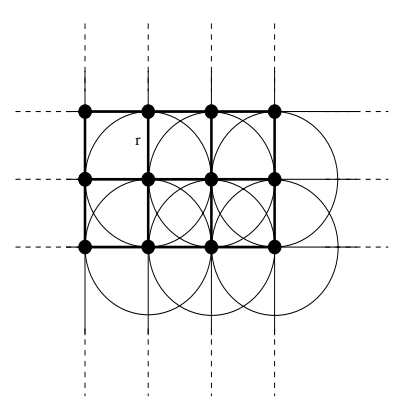

Figure 9: SQR: Square-grid based topology. (The bold dots denote the active sensor nodes. The bold straight lines are the edges of the connectivity graph. The circles denote the coverage/connectivity radii.)

that we discuss here can be considered as practical adaptations of the wakeup based cc-topology construction strategies considered before, while one of them is motivated by some key insights in the analysis presented earlier. These wakeup algorithms can be implemented in a fully distributed manner, with zero or low coordination between the sensor nodes. Table 1 shows the level of coordination and distance/direction information requirements for each wakeup algorithm described here.

In this section we present four different algorithms to construct an efficient cc-topology. In order to compute multiple, independent cc-topologies for redundancy and fault-tolerance we iteratively run each of these algorithms on the remaining set of nodes, after eliminating the nodes that have been placed in previously computed cc-topologies. Our experimental results show that the number of such independent cc-topologies computed by the best of these algorithms approach to within $70 \%$ of a loose upper bound of the optimal number of topologies achievable.

\subsection{Approx-Random}

The approx-random ( $a-R A N D)$ algorithm is a distributed approximation of the random topology construction algorithm described 


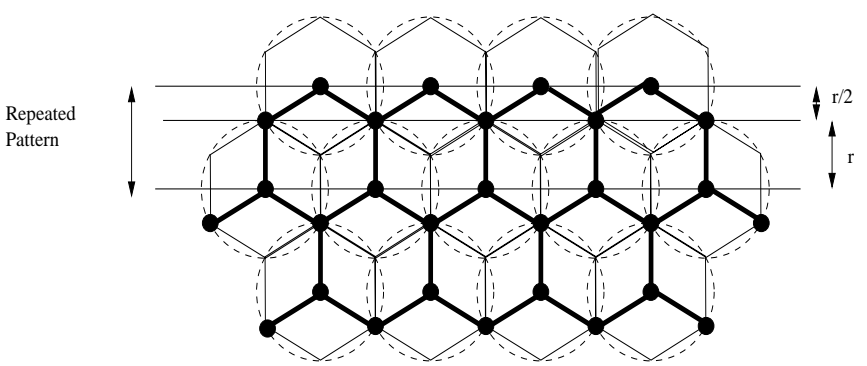

Figure 10: HEX: Hexagonal-grid based topology. (The bold dots denote the sensor nodes. The bold straight lines are the edges of the connectivity graph. The circles denote the coverage/connectivity radii.)

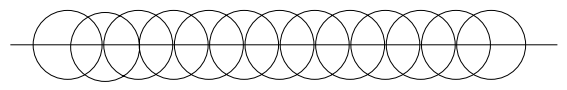

(a)

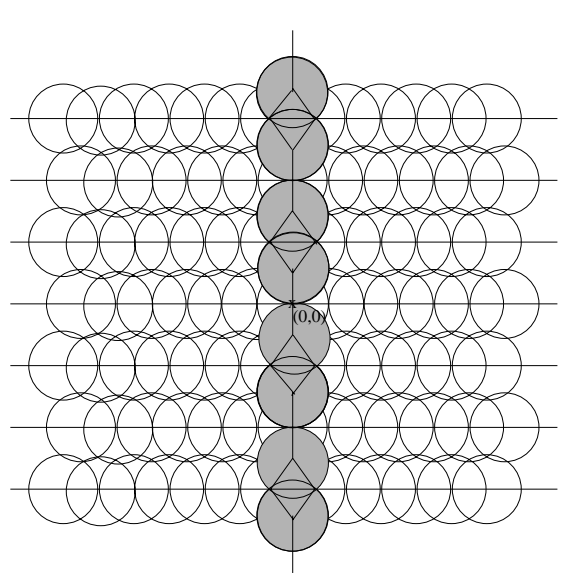

(b)

Figure 11: (a) A strip, (b) STR: A connected strip-based topology. (The bold dots denote the active sensor nodes. The bold straight lines are the edges of the connectivity graph. The circles denote the coverage/connectivity radii.)

in Section 3.1. Let us assume that the spatial density of the deployed sensors is $d$. Thus, if $N$ sensors are deployed in a region of dimension $D \times D$, then $d=\left(N / D^{2}\right)$. Also assume that the targeted spatial density of active nodes in $\lambda$. Based on our observations in Section 3.1, we choose $\lambda$ between $\frac{1.6}{r^{2}}$ and $\frac{1.9}{r^{2}}$, Then, in the a-RAND algorithm, in any iteration, each node in the network should wake up independently with probability $(p=\lambda / d)$. Such a choice of $p$ is adequate to give good performance in dense networks as shown in Section 3. However in less dense networks, through simulations we have found that its prudent to use more conservative (higher) values of $p$. This is because the required probability of active nodes to construct cc-topologies is based on an uniform distribution of nodes within the region of interest, which intuitively holds in very dense networks. However, the lower the density of sensor deployment, the less uniform the consequent node distribution is. Hence we choose a conservative (higher) wakeup probability, $p^{\prime}$. In general the choice of this conservative estimate should depend on the actual spatial density of deployed nodes. By choosing this higher value for $p^{\prime}$ we increase the number of nodes in each cc-topology which can negatively impact the performance of

\begin{tabular}{|c|c|c|c|}
\hline Algorithm & $\begin{array}{c}\text { Level of } \\
\text { coordination }\end{array}$ & $\begin{array}{c}\text { Distance } \\
\text { information } \\
\text { required ? }\end{array}$ & $\begin{array}{c}\text { Sense of } \\
\text { direction } \\
\text { required ? }\end{array}$ \\
\hline a-RAND & Zero & No & No \\
\hline a-HEX & Low & Yes & Yes \\
\hline a-STR & Low & Yes & Yes \\
\hline c-BFS & Low & Yes & No \\
\hline
\end{tabular}

Table 1: Comparative requirements and complexity of the four algorithms.

our proposed scheme. Through simulations (presented later in this section) over different low deployment densities that we considered we found that a choice of $p^{\prime}$ which was 1.5 times the value of $p$ was adequate for good performance.

Based on the value of $p^{\prime}$ the number of nodes thus woken up contribute to one connected-covered set. This process can be repeated to form multiple connected-covered sets, between which the sensing activity can be time-shared. Note that the node wakeup procedure of the a-RAND algorithm do not require the individual sensor nodes to know their absolute or relative location or any other information regarding the network topology, and can therefore be implemented with no coordination. This a very attractive feature of the algorithm, from the viewpoint of practical implementation.

\subsection{Approx-STR and Approx-HEX}

The approx-STR ( $a$-STR) and the approx-HEX ( $a$-HEX) are practical approximations to the STR and HEX topology solutions described in Section 3.2. Recall that we constructed the STR and HEX topologies under the assumption that the network is so dense that there is a node close to any chosen location, and so we could activate a sensor node at any location of our choice. However, in a realistic setting, this is not exactly true, particularly if the sensor network is only moderately dense. In the a-STR and a-HEX solutions, we try to approximate the STR and HEX topologies as closely as possible. Here we try to wakeup nodes according to the STR and HEX topologies, but if a node is not available as the precise chosen location, we pick a node that is closest to that location, provided that the node is within a conservative estimate of the communication radius. This conservative estimate, $r^{\prime}$ is set to be $r(1-\epsilon)$. We make such a choice of $r^{\prime}$ for two reasons: (1) Realizing that in practical wireless environments, wireless signals are not isotropic and instead attenuate unequally in different directions. Hence such a conservative estimate helps us deal with irregularities of the communication range. (2) In less dense networks, if the a-HEX and a-STR algorithms decide to optimize choice of nodes such that they are exactly $r$ apart, then a slight shift in the actual location of nodes will cause disconnection. Note that by choosing higher values of $\epsilon$ the performance, each scheme needs to find more nodes to construct each cc-topology, thereby reducing the total number of cc-topologies. In our simulations (discussed later in this section) we observed that choosing $\epsilon=0.2$ is an adequate value to construct multiple good cc-topologies. Similarly, in the a-STR scheme, instead of choosing a single vertical strip to guarantee connectedness of each topology, we use multiple such strips $(\leq 10)$ to provide redundancy in face of small random shifts between expected and actual locations of the sensors. Such a choice is redundant in dense networks but is useful in the less dense scenarios.

Note that the wakeup policies in this case require a sensor node to know its location. Specifically, since nodes are woken up based on their proximity to a precalculated location, information about dis- 
tance between neighboring nodes, as well as a globally consistent sense of direction, are needed. The location information can be obtained if the nodes have access to GPS, or the relative location can be computed using some distributed localization algorithm. Also note that this algorithm can easily be implemented in a distributed manner since the node closest to a chosen location can be found distributedly by having the nodes exchange some information with its local neighborhood. However, these algorithms clearly require more information and more coordination than the a-RAND algorithm.

\subsection{Constrained-BFS}

The constrained-BFS ( $c-B F S$ ) algorithm is a deterministic breadthfirst-search (BFS) based algorithm which is developed using the intuition obtained from the analysis of the lower bound, as outlined in Section 2. In c-BFS, we traverse the connectivity graph of the sensor network using BFS (we could use a depth-first-search (DFS) traversal technique as well). When we visit a node as part of the BFS procedure, we wake up that node if the new area covered by that node (i.e., the area covered by that node that is not already covered by nodes that have already woken up) is no less than $\alpha$ $1.914 r^{2}$; otherwise, the node is put to sleep again. $\alpha$ is a value between 0 and 1 and defines the "aggressiveness" of the algorithm in adding nodes and a value of 0.5-0.6 is adequate for most purposes (as observed through simulations). Note that the use of the term $1.914 r^{2}$ is motivated by the fact that in the derivation of the lower bound in Section 2, the new area covered by each additional sensor is bounded by $1.914 r^{2}$. If the node is woken up, then its neighbors (that haven't already woken up) are added to the BFS queue (so that they are visited later by the BFS algorithm); otherwise, the neighboring nodes are not added to the BFS queue. Since BFS can be executed in a distributed manner using only local information exchange amongst nodes, the c-BFS algorithm can be implemented in a distributed manner as well. By imposing this minimum restriction on the "new" area that a potential member of the current topology must cover, we ensure that the nodes in the topology are well spaced, while being connected. To implement c-BFS, a node only needs to know its distance from its neighboring nodes. Note that no globally consistent sense of direction is needed, since a node is selected to be awake based only on the new area it covers.

\subsection{Simulation Results}

In all of the simulations, sensor nodes are deployed uniformly at random in an area of size $1000 \times 1000$. As discussed in Section 3, targeting perfect connected coverage of the entire region of interest leads to significant increase in required densities of sensor deployment. Hence as explained in that section, for reasons of efficiency in sensor deployment we target a connected-coverage fraction of upto $90 \%$ of the area of interest by each cc-topology constructed by the algorithms, i.e., we require that at least $90 \%$ of the region of interest is connected and covered by each cc-topology generated. We show through simulations that our proposed algorithms are efficient under such connected-coverage requirements.

As explained before, each of the algorithms are run iteratively, in each iteration a single cc-topology is found and the corresponding nodes are removed before running the next iteration. The algorithm terminates when all nodes have been exhausted. The simulation terminates when there are no more nodes left which can be taken into consideration to form a cc-topology.

The comparative performance of the four algorithms in terms of the number of cc-sets found is given in Table 2. The column "upper bound" is a loose upper bound on the number of cc-topologies (or cc-sets) that can be computed by any solution, derived from

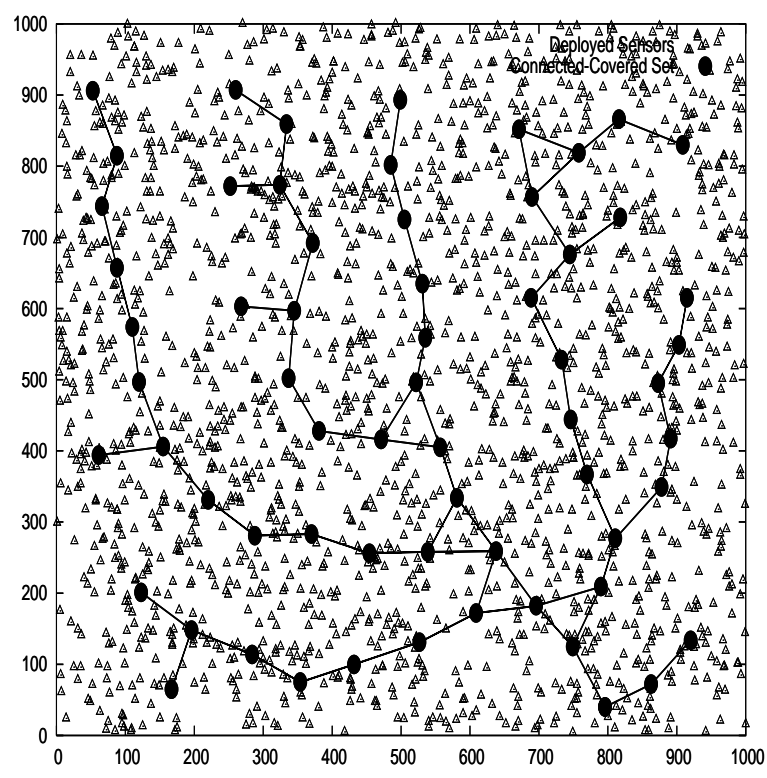

Figure 12: Example: Connected-covered set formed by c-BFS. The small triangles represent the deployed sensors, while the shaded circles represent the sensors included in the cc-topology in a single run of c-BFS. This topology provides greater than $90 \%$ of the region of interest and, the bold lines represent the edges of the connectivity graph.

our results in Section 2, and is equal to $\frac{1.91 N r^{2}}{D^{2}}$, where $N$ is the number of nodes deployed, $r(=100)$ is the coverage/connectivity radius, and $D(=1000)$ is the dimension of the deployment region. The column "\# of cc sets found" lists the number of cc-topologies found by running our algorithm iteratively. The column "perf. ratio" shows the performance of our algorithm (in terms of computing cc-topologies) with respect to the upper bound. Thus perf. ratio $=$ (\# of cc sets found / upper bound).

The tables show that the pattern-based schemes (a-RAND, aSTR and a-HEX) attain about $30 \%$ performance with respect to the upper bound. (Note here that for most general networks, the upper bound is not very tight, and so the performance of these algorithms with respect to the optimal will typically be much better). Amongst these a-STR performs the best. We also observe that $c$ BFS performs much better than the other algorithms, and attains a performance of about $70 \%$ with respect to the upper bound. Figure 12 shows the cc-topology formed from a sample run of the cBFS algorithm.

Table 3 shows the sensitivity of the performance of our algorithms with respect to the different parameters associated with them. Note that the parameters $p^{\prime}, \epsilon, \alpha$ are associated with the a-RAND, a-STR and c-BFS algorithms respectively. In the simulation results shown in 2, the values of the parameters were chosen so as to yield the best performance. These values are shown in bold in the table. The table shows how the performance degrades as the the parameters are perturbed from their optimal values. We choose $n=2000$ as an example; simulation results for other values of $n$ showed similar trends.

\section{RELATED WORK}

The use of alternating sleep and wakeup cycles to improve lifetime of wireless networks have been explored in a number of recent literature including GAF [12], SPAN [10], STEM [14], AFECA [13], LEACH [11]. However the goal of these recent research activities 
Table 2: Performance Comparison of Heuristics

\begin{tabular}{|c|c|c|c|c|c|c|c|c|c|}
\hline & & \multicolumn{2}{|c|}{ a-RAND } & \multicolumn{2}{c|}{ a-HEX } & \multicolumn{2}{c|}{ a-STR } & \multicolumn{2}{c|}{ c-BFS } \\
\hline \# nodes & $\begin{array}{c}\text { upper } \\
\text { bound }\end{array}$ & $\begin{array}{c}\text { \# cc sets } \\
\text { found }\end{array}$ & $\begin{array}{c}\text { perf. } \\
\text { ratio }\end{array}$ & $\begin{array}{c}\text { \# cc sets } \\
\text { found }\end{array}$ & $\begin{array}{c}\text { perf. } \\
\text { ratio }\end{array}$ & $\begin{array}{c}\text { \# cc sets } \\
\text { found }\end{array}$ & $\begin{array}{c}\text { perf. } \\
\text { ratio }\end{array}$ & $\begin{array}{c}\text { \# cc sets } \\
\text { found }\end{array}$ & $\begin{array}{c}\text { perf. } \\
\text { ratio }\end{array}$ \\
\hline 200 & 4 & 1 & 0.25 & 1 & 0.25 & 1 & 0.25 & 1 & 0.25 \\
500 & 10 & 2 & 0.20 & 2 & 0.20 & 2 & 0.20 & 3 & 0.30 \\
1000 & 19 & 4 & 0.21 & 5 & 0.26 & 5 & 0.26 & 8 & 0.42 \\
2000 & 39 & 10 & 0.25 & 10 & 0.25 & 11 & 0.28 & 22 & 0.56 \\
4000 & 76 & 25 & 0.328 & 21 & 0.27 & 23 & 0.30 & 48 & 0.63 \\
6000 & 115 & 35 & 0.30 & 32 & 0.27 & 36 & 0.31 & 76 & 0.66 \\
8000 & 153 & 48 & 0.31 & 42 & 0.27 & 49 & 0.32 & 106 & 0.69 \\
10000 & 192 & 61 & 0.31 & 53 & 0.27 & 64 & 0.33 & 134 & 0.70 \\
\hline
\end{tabular}

Table 3: Sensitivity Analysis

\begin{tabular}{|c|c|c|c|c|c|}
\hline \multicolumn{2}{|c|}{$p^{\prime}$} & \multicolumn{2}{c|}{$\epsilon$} & \multicolumn{2}{c|}{$\alpha$} \\
$n=2000$ & \multicolumn{2}{c|}{$n=2000$} & \multicolumn{2}{c|}{$n=2000$} \\
\hline Value & Perf. & Value & $\begin{array}{c}\text { Perf. } \\
\text { Ralio }\end{array}$ & Value & $\begin{array}{c}\text { Perf. } \\
\text { Ratio }\end{array}$ \\
\hline & Ratio & & Ratio & & 0.44 \\
0.075 & 0.20 & 0.1 & 0.13 & 0.4 & 0.51 \\
0.1 & 0.23 & 0.15 & 0.23 & 0.5 & 0.5 \\
$\mathbf{0 . 1 2 5}$ & $\mathbf{0 . 2 5}$ & $\mathbf{0 . 2}$ & $\mathbf{0 . 2 8}$ & $\mathbf{0 . 6 0}$ & $\mathbf{0 . 5 6}$ \\
0.15 & 0.20 & 0.25 & 0.25 & 0.7 & 0.46 \\
0.175 & 0.15 & 0.3 & 0.23 & 0.8 & 0.41 \\
\hline
\end{tabular}

has been to construct-connected topologies only, while we consider the problem of connected-coverage in this paper. Research on coverage and connectivity has a long history [4, 6, 3], although most of the existing literature address coverage and connectivity issues separately. However, there have been recent attempts to integrate these issues, and study its applications in the context of sensor networks. The work most closely related to ours is by Gupta et.al. [9], where they consider the problem of constructing a single cc-topology in multi-hop wireless networks. The work presented in this paper differs from theirs in approach, objectives, and results, as described in Section 1. Booth et.al. [1] investigate the coverage and percolation properties of disks placed to cover a given set of points (as opposed to a given region). The authors in Shakkottai et.al. [2] assume that the nodes are already placed along a square grid, and are interested in finding the critical transmission/sensing radius (in the asymptotic regime) that ensures connectivity and/or coverage. Although our paper looks at connectivity and coverage in an integrated manner, our problem definition and research objectives are very different from those in $[1,2]$. Instead of studying the asymptotic properties of connectivity and coverage, we are interested in finding multiple cc-topologies efficiently from a densely deployed sensor network. Although the authors in [2] address both connectivity and coverage, the system model, problem definition and research objectives of that work is very different from our proposed work. More specifically, the authors in [2] assume that the nodes are already placed along a square grid, and are interested in finding the critical transmission/sensing radius that ensures connectivity and/or coverage. Moreover, note that the results in these works are asymptotic in nature, and may not be applicable to the optimal deployment question where we are interested in the non-asymptotic regime. The authors in [15] present protocols to achieve guaranteed degrees of coverage and connectivity, and a geometric analysis of the relationship between coverage and connectivity. While we also use geometric arguments in this paper, our objectives are different. Our goal in this paper is to find as many independent cc-topologies of sensor nodes as possible. The detailed implementation of these algorithms in the form of protocols is not addressed here. In [18], the authors also consider the problem of minimizing the number of active nodes, which providing a desired coverage. However, our work differs from [18] in two main aspects. We focus on deriving the optimal density of nodes needed to provide connected-coverage, and the intuition gained from the analysis is used in developing algorithms that come close to the optimal operation. Further, our work also focuses on finding and maximizing the actual number of independent cc-topologies in the network. In [16], the authors consider the problems of power assignment and network lifetime in sensor networks. In their formulation, the objective is to maximize the lifetime subject to the battery power constraints for each node. The nodes may have different battery levels and belong to multiple directed spanning graphs. In this paper, all nodes are assumed to have the same battery levels, and sets of nodes which provide coverage to the region under consideration (all the nodes in one set form a connected graph) do not have nodes in common. Our focus is on to develop simple but efeective heuristics which can be deployed easily in actual sensor networks. Moreover, [16] uses a transmission range approach, i.e. changing transmission power while still maintaining connectivity and coverage, while this paper uses a sleep scheduling approach, i.e. nodes not involved currently in sensing sleep in order to conserve power. In [17] the authors propose an explicit sleep/wake-up schedule to maximize lifetime of the network. Unlike a number of other protocols, this paper provides rigid theoretical performance guarantees.

\section{CONCLUDING REMARKS}

We present simple low-coordination wakeup-based cc-topology construction schemes that perform well in a wide range of sensor deployment scenarios (different densities). We use geometric arguments to provide necessary performance bounds in each case. While these geometric arguments assume regular communication structures (e.g., a circular range) we believe that these results are applicable even in many practical network scenarios. For example, by making conservative estimates of communication ranges we can tide over some of the irregularities of different communication ranges of a sensor node in different directions while taking some performance penalty. We propose such an approach in our cctopology computation algorithms. Additionally, we are also continuing to study effect of various irregular communication ranges to our proposed algorithms and how the latter can be better adapted to such scenarios. 


\section{REFERENCES}

[1] L. Booth, J. Bruck, M. Franceschetti and R. Meester, "Covering Algorithms, Continuum Percolation, and the Geometry of Wireless Networks," Annals of Applied Probability, 13(2), May 2003.

[2] S. Shakkottai, R. Srikant, N. Shroff,"Unreliable Sensor Grids: Coverage, Connectivity and Diameter," To appear in the Proc. IEEE Infocom, San Francisco, March 2003.

[3] P. Gupta and P. R. Kumar, "Critical Power for Asymptotic Connectivity in Wireless Networks," pp. 547-566, in Stochastic Analysis, Control, Optimization and Applications: A Volume in Honor of W.H. Fleming. Edited by W.M. McEneany, G. Yin, and Q. Zhang, Birkhauser, Boston, 1998.

[4] R. Kershner, "The number of circles covering a set", American Journal of Mathematics, 61:665-671, 1939.

[5] W. Kuperberg, "Packing and covering problems in two and three dimensions", Alabama Journal of Mathematics 20, 1996.

[6] P. Hall, Introduction to the Theory of Coverage Processes, John Wiley \& Sons, 1988.

[7] D. S. Hochbaum and W. Maas, "Approximation schemes for covering and packing problems in image processing and VLSI", J. ACM, 32:130-136, 1985.

[8] J. Quintanilla, S. Torquato, and R. Ziff, "Efficient Measurement of the Percolation Threshold for Fully Penetrable Disks", Journal of Physics A: Mathematics and General, 33, L399-L407 (2000).

[9] H. Gupta, S. Das, Q. Gu, "Connected Sensor Cover: Self-Organization of Sensor Networks for Efficient Query Execution", Proc. ACM MobiHoc, June 2003.
[10] B. Chen, K. Jamieson, H. Balakrishnan, R. Morris, ”Span: An Energy-Efficient Coordination Algorithm for Topology Maintenance in Ad Hoc Wireless Networks", Proc. ACM Mobicom, July 2001.

[11] W. Heizelman, A. Chandrakasan, ”Energy-efficient Communication Protocols for Wireless Microsensor Networks", Proc. HICSS, Jan. 2000.

[12] Y. Xu, J. Heidemann, D. Estrin, ”Geography-Informed Energy Conservation for Ad-Hoc Routing", Proc. ACM Mobicom, July 2001.

[13] Y. Xu, J. Heidemann, D. Estrin, "Adaptive Energy Conserving Routing for Multihop Ad Hoc Networks", Tech. Report. 527, USC/ISI, Oct. 2000.

[14] C. Schurgers, V. Tsiatsis, S. Ganeriwal, M. Srivastava, "Topology Management for Sensor Networks: Exploiting Latency and Density", Proc. ACM MobiHoc, June 2002.

[15] Xiaorui Wang, Guoliang Xing, Yuanfang Zhang, Chenyang Lu, Robert Pless, Christopher Gill, "Integrated Coverage and Connectivity Configuration in Wireless Sensor Networks", Proc. ACM Sensys, November 2003.

[16] G. Calinescu, S. Kapoor, A. Olshevsky, A. Zelikovsky, "Network Lifetime and Power Assignment in Ad-Hoc Wireless Networks", Proc. ESA, 2003.

[17] T. Moscibroda, R. Wattenhoffer, "Maximizing the Lifetime of Dominating Sets", Proc. WMAN, 2005

[18] H. Zhang, J.C. Hou, "Maintaining Sensing Coverage and Connectivity in Large Sensor Networks", Technical Report UIUCDCS-R-2003-2351, June 2003. 